\title{
Asymptotic enumeration on self-similar graphs with two boundary vertices
}

\author{
Elmar Teufl ${ }^{1}$ and Stephan Wagner ${ }^{2} \|$ \\ ${ }^{1}$ Fakultät für Mathematik, Universität Bielefeld, P.O.Box 100131, 33501 Bielefeld, Germany \\ ${ }^{2}$ Department of Mathematical Sciences, Stellenbosch University, Private Bag X1, Matieland 7602, South Africa
}

received April 9, 2008, revised December 23, 2008, accepted December 23, 2008.

\begin{abstract}
We study two graph parameters, namely the number of spanning forests and the number of connected subgraphs, for self-similar graphs with exactly two boundary vertices. In both cases, we determine the general behavior for these and related auxiliary quantities by means of polynomial recurrences and a careful asymptotic analysis. It turns out that the so-called resistance scaling factor of a graph plays an essential role in both instances, a phenomenon that was previously observed for the number of spanning trees. Several explicit examples show that our findings are likely to hold in an even more general setting.
\end{abstract}

Keywords: spanning forests, connected subgraphs, self-similar graphs, asymptotic enumeration

\section{Introduction}

In several recent papers [3, 4, 5, 6, 14, 15, 16] the enumeration of various graph-theoretical objects on selfsimilar graphs was studied, including, among others, the number of spanning trees and forests, matchings, and connected subgraphs. Some of these counting problems have a background in theoretical physics, as also explained in [3]: the number of spanning forests of a graph can be seen as a special value of the Tutte polynomial $T(G, x, y)$ of a graph (namely, the value for $x=2, y=1$ ). On the other hand, it also occurs as a special $q \rightarrow 0$ limit of the partition function of the $q$-state Potts model, see for example [13]. While this and related problems are well-studied for lattices, [3] is the first work where fractal-type structures are investigated. There, the authors were concerned with the asymptotic behavior of the number of spanning forests $\left|\operatorname{SF}\left(G_{n}\right)\right|$ for an increasing family of graphs $G_{n}$, and in particular with the associated asymptotic growth constant

$$
\lim _{n \rightarrow \infty} \frac{\log \left|\operatorname{SF}\left(G_{n}\right)\right|}{\left|G_{n}\right|}
$$

which is a quantity of physical interest. The authors of [3] were able to calculate numerical values for the growth constants of Sierpiński graphs with small dimension. In the present paper, we study the problem

\footnotetext{
$\dagger^{T}$ This material is based upon work supported by the German Research Foundation DFG under grant number 445 SUA-113/25/0-1 and the South African National Research Foundation under grant number 65972.
}

1365-8050 @ 2009 Discrete Mathematics and Theoretical Computer Science (DMTCS), Nancy, France 
in more generality, but we have to restrict ourselves to a special type of construction that will be explained in the following section. The other counting problem that we address is the enumeration of connected subgraphs. We will see that similar techniques can be used for both problems and that the so-called resistance scaling factor of a graph with respect to two of its vertices plays a crucial role in both instances. This phenomenon also occurs in the enumeration of spanning trees, which was exhibited in [16].

Let us demonstrate this in the case of the aforementioned sequence of finite Sierpiński graphs which are depicted on Figure 1 . The number of spanning trees in the level- $n$ graph $X_{n}$ is described by a system
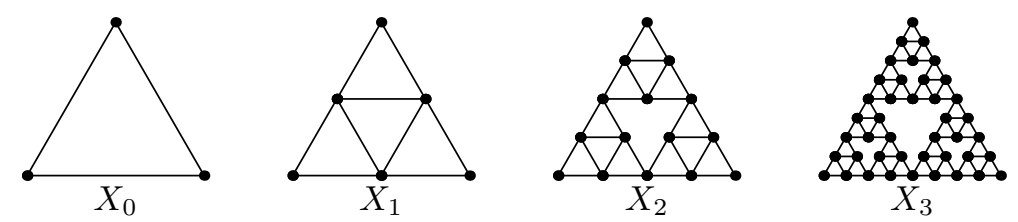

Fig. 1: Finite Sierpiński graphs $X_{0}, X_{1}, X_{2}, X_{3}$.

of polynomial recurrence equations:

$$
\begin{aligned}
& a_{1, n+1}=6 a_{1, n}^{2} a_{2, n}, \\
& a_{2, n+1}=7 a_{1, n} a_{2, n}^{2}+a_{1, n}^{2} a_{3, n}, \\
& a_{3, n+1}=14 a_{2, n}^{3}+12 a_{1, n} a_{2, n} a_{3, n},
\end{aligned}
$$

where $a_{k, n}$ is the number of spanning forests in $X_{n}$ with $k$ components each of which contains exactly one "boundary vertex" of $X_{n}$, see [6, 14]. From this it is not too difficult to derive an explicit formula for the number $\tau\left(X_{n}\right)$ of spanning trees in $X_{n}$ :

$$
\tau\left(X_{n}\right)=\sqrt[4]{\frac{3}{20}}\left(\frac{5}{3}\right)^{-n / 2}(\sqrt[4]{540})^{3^{n}} .
$$

In [16] it was proved that the number of spanning trees can be computed explicitly for a large class of self-similar graphs with a high degree of symmetry. Similarly, the number of connected subgraphs or connected subsets can be described by a system of seven polynomial recurrence equations:

$$
\begin{aligned}
\boldsymbol{b}_{\mathbf{1 , n + 1}} & =\mathbf{1 2} \boldsymbol{b}_{\mathbf{1}, \boldsymbol{n}} \boldsymbol{b}_{\mathbf{2}, \boldsymbol{n}} \boldsymbol{b}_{\mathbf{4}, \boldsymbol{n}}+\mathbf{1 4} \boldsymbol{b}_{\mathbf{2}, \boldsymbol{n}}^{\mathbf{3}}+3 b_{1, n} b_{5, n}^{2}+\cdots, \\
\boldsymbol{b}_{\mathbf{2}, \boldsymbol{n}+\mathbf{1}} & =\boldsymbol{b}_{\mathbf{1}, \boldsymbol{n}} \boldsymbol{b}_{\mathbf{4}, \boldsymbol{n}}^{\mathbf{2}}+\mathbf{7}_{\mathbf{2}, \boldsymbol{n}}^{\mathbf{2}} \boldsymbol{b}_{\mathbf{4}, \boldsymbol{n}}+b_{2, n} b_{4, n}^{2}+3 b_{2, n} b_{5, n}^{2}+\cdots, \\
b_{3, n+1} & =2 b_{1, n} b_{4, n} b_{5, n}+\cdots, \\
\boldsymbol{b}_{\mathbf{4}, \boldsymbol{n}+\mathbf{1}} & =\mathbf{6} \boldsymbol{b}_{\mathbf{2}, \boldsymbol{n}} \boldsymbol{b}_{\mathbf{4}, \boldsymbol{n}}^{\mathbf{2}}+b_{4, n}^{3}+3 b_{4, n} b_{5, n}^{2}, \\
b_{5, n+1} & =4 b_{2, n} b_{4, n} b_{5, n}+\cdots, \\
b_{6, n+1} & =2 b_{2, n} b_{5, n}^{2}+\cdots, \\
b_{7, n+1} & =3 b_{3, n} b_{5, n}^{2}+\cdots .
\end{aligned}
$$

We refer to [15] for details, including a precise definition of the quantities involved. It turns out that the asymptotically significant terms are those which are written in bold. We point out that this part of the 
system agrees with the system for spanning trees. Hence the number of connected subgraphs (or subsets) is asymptotically given by

$$
c \cdot\left(\frac{5}{3}\right)^{-\frac{n}{2}} \cdot \beta^{3^{n}}
$$

for constants $c=6.163424 \ldots$ and $\beta=2.3032106556 \ldots$ In [15] it was conjectured that a similar formula holds for other self-similar graphs as well, which we will verify for a very specific class in this paper, namely self-similar graphs with two "boundary" vertices. We obtain precise asymptotic information for the number of spanning forests and the same for the number of connected subgraphs under mild conditions on the geometry of $X_{n}$.

\section{Setting and statement of results}

Let $G=(V G, E G)$ be a finite (multi-)graph with vertex set $V G$ and edge set $E G$. Fix two distinguished vertices $v, w$ in $G$. Throughout this work we denote by $s=|E G|$ the number of edges in $G$, by $d=$ $d_{G}(v, w)$ the distance of the distinguished vertices $v$ and $w$ in $G$, and by $\delta=|V G|-1$ the number of edges in a spanning tree of $G$. Using $G$ as model, construct a sequence $X_{0}, X_{1}, X_{2}, \ldots$ of self-similar (multi-)graphs as follows:

- The initial graph $X_{0}$ is given together with two distinguished vertices $v_{0}, w_{0}$ and is assumed to be finite.

- The graph $X_{n+1}$ is obtained by replacing every edge of $G$ by an isomorphic copy of $X_{n}$, where $v_{n}, w_{n}$ are used for linkage. There are two possibilities for each replacement. Fix one of these once and forever. The distinguished vertices $v_{n+1}, w_{n+1}$ of $X_{n+1}$ emerge from $v, w$ in $G$.

For an edge $e \in E G$ we write $\mathrm{h}_{v}(e) \in V G$ for the endpoint of $e$ which is merged with $v_{n}$ during the construction and call the vertex $\mathrm{h}_{v}(e)$ the $v$-end of the edge $e$. We define the $w$-end $\mathrm{h}_{w}(e)$ of $e$ analogously. Thus, in every stage of the construction an edge $e$ in $G$ is replaced by $X_{n}$, so that the vertex $\mathrm{h}_{v}(e)$ is merged with $v_{n}$ and $\mathrm{h}_{w}(e)$ is merged with $w_{n}$.

We always assume that $X_{0}$ and $G$ are connected, so that the graphs $X_{n}$ are connected, too. By considering edges of $G$ as resistors with unit resistance we may regard $G$ as a electrical network. With respect to the boundary $v, w$ (source, sink) the network $G$ is electrically equivalent to a single resistor with some resistance $\rho$, which is called the resistance scaling factor of $G$ with respect to $v$ and $w$, see for example [1, 8, 12, 15]. Notice that $\rho \leq 1$ if $d=d_{G}(v, w)=1$.
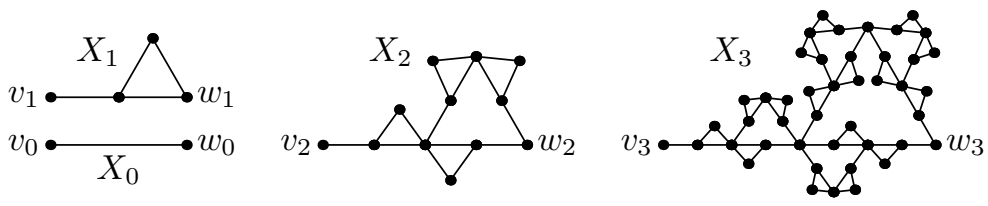

Fig. 2: The Austria graphs $X_{0}, X_{1}, X_{2}$, and $X_{3}$.

Example. The "Austria" graphs $X_{0}, X_{1}, \ldots$ were first studied in [10] with respect to volume growth and later also in [15] in the context of combinatorial enumeration (their shape resembles a map of Austria, see Figure 2). Since the initial graph is given by $X_{0}=K_{2}$, the model graph $G$ equals $X_{1}$. The orientation of the replacement can be seen in $X_{2}$ and $X_{3}$ in Figure 2. Obviously, $s=4, d=2, \delta=3$, and $\rho=\frac{5}{3}$. 
Example. In [11] spectral properties of the modified Koch curve were studied. It is a minor but interesting variation of the fractal Koch curve. The first few graphs in the associated graph sequence are depicted in Figure 3 Since $X_{0}=K_{2}$ the model graph $G$ is again $X_{1}$. Due to symmetry there are no choices during the replacement.

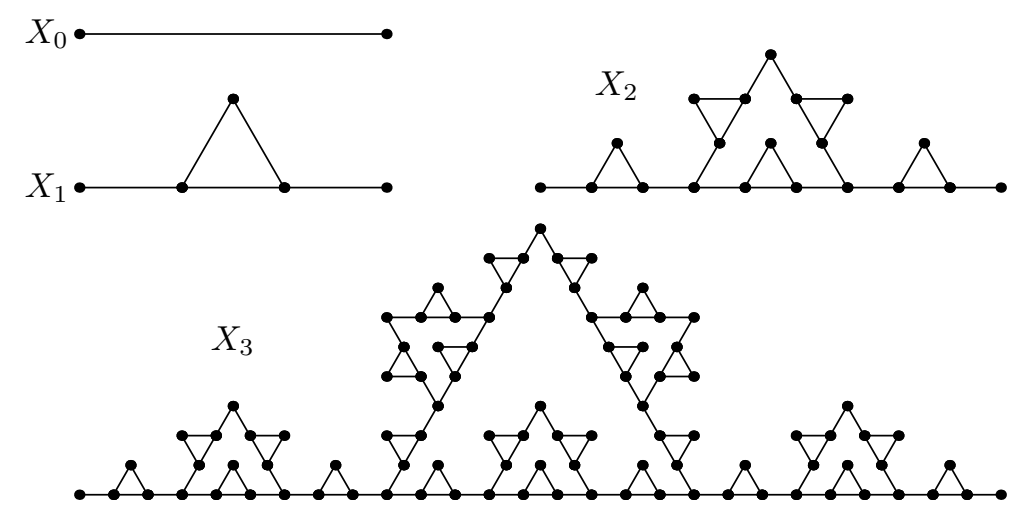

Fig. 3: The finite modified Koch graphs $X_{0}, X_{1}, X_{2}$, and $X_{3}$.

Theorem 1. Assume that $s>1$, then two different cases concerning the asymptotics of spanning forests in $X_{n}$ may occur:

(I) The number of spanning forests in $X_{n}$ which do not connect $v_{n}$ and $w_{n}$ is given by

$$
C_{1} \beta_{1}^{s^{n}}(1+o(1))
$$

whereas the number of spanning forests in $X_{n}$ connecting $v_{n}$ and $w_{n}$ satisfies

$$
C_{2} \beta_{1}^{s^{n}} \beta_{2}^{-d^{n}}(1+o(1))
$$

for certain constants $\beta_{1}>1, \beta_{2} \geq 1, C_{1}>0, C_{2}>0$.

(II) The number of spanning forests in $X_{n}$ which do not connect $v_{n}$ and $w_{n}$ is given by

$$
C_{3} \rho^{\frac{\delta-1}{s-1} n} \beta_{3}^{s^{n}}(1+o(1)),
$$

whereas the number of spanning forests in $X_{n}$ connecting $v_{n}$ and $w_{n}$ satisfies

$$
C_{4} \rho^{-\frac{s-\delta}{s-1} n} \beta_{3}^{s^{n}}(1+o(1))
$$

for certain constants $\beta_{3}>1, C_{3}>0, C_{4}>0$.

Expressions for these constants are provided in the proof. Depending on the values of $\rho$ and $d$, we get the following description:

- If $\rho \geq 1$ then Case $\square$ occurs. 
- If $\rho<1$ and $d=1$ then Case II occurs.

- If $\rho<1$ and $d>1$ then both cases are possible depending on the initial graph $X_{0}$. Here it may happen that the term $\beta_{1}^{s^{n}}(1+o(1))$ of Case $\left[\right.$ must be replaced by the weaker term $\beta_{1}^{s^{n}+O(1)}$ (this situation is described in the proof).

The proof and an example for the case $\rho<1$ and $d>1$ are provided in Section 3

Theorem 2. Assume that $s>1$, that $v, w$ are leaves (i.e. vertices with degree 1 ) in $G$, that $v_{0}, w_{0}$ are leaves in $X_{0}$, and that $G$ is not a path, then there are constants $\beta_{4}>1$ and $C_{5,0}>0, C_{5,1}>0$, so that the number of non-empty connected subgraphs of $X_{n}$ is given by

$$
C_{5, k} \rho^{-\frac{s-\delta}{s-1} n} \beta_{4}^{s^{n}}\left(1+O\left(2^{-n}\right)\right),
$$

where $k \in\{0,1\}$ is the remainder of the division of $n$ by 2 .

The assumption that $v, w$ and $v_{0}, w_{0}$ are leaves assures that the maximal degree $X_{n}$ is uniformly bounded. Together with $s>1$ it implies further that $\rho \geq 2$. In the case that $G$ is a path it is easy to derive an exact formula for the number of non-empty connected subgraphs of $X_{n}$. Theorem 2 is proved in Section 4

\section{Spanning forests}

This section is devoted to the proof of Theorem 1. Thus we always assume that $s>1$. Let $\operatorname{SF}(G)$ be the set of all spanning forests in $G$. Introduce auxiliary sets: $\mathrm{SF}^{\bullet}(G), \mathrm{SF}^{\circ}(G)$. The first set contains all spanning forests of $G$ connecting $v$ and $w$, whereas the latter denotes the set of all spanning forests of $G$, where $v$ and $w$ are not connected by the forest. Denote by $\operatorname{SF}_{m}(G)$ the set of all spanning forests of $G$ with $m$ edges and combine these notations in the obvious way, e.g. $\mathrm{SF}_{m}^{\circ}(G)$. Finally, we use $\mathrm{SF}\left(X_{n}\right), \mathrm{SF}^{\bullet}\left(X_{n}\right), \mathrm{SF}_{m}\left(X_{n}\right)$ analogously.

We are interested in the number $\left|\operatorname{SF}\left(X_{n}\right)\right|$ of spanning forests in $X_{n}$. Obviously, the disjoint union $\mathrm{SF}^{\circ}\left(X_{n}\right) \uplus \mathrm{SF}^{\bullet}\left(X_{n}\right)=\operatorname{SF}\left(X_{n}\right)$ is the set of all spanning forests of $X_{n}$. For simplicity, set $a_{n}=$ $\left|\mathrm{SF}^{\bullet}\left(X_{n}\right)\right|, b_{n}=\left|\mathrm{SF}^{\circ}\left(X_{n}\right)\right|$. Notice that the sequence $b_{n}$ tends to infinity since $s>1$. Since every spanning forest of $X_{n+1}$ can be decomposed into spanning forests on each copy of $X_{n}$, we obtain the recursions

$$
\begin{aligned}
& a_{n+1}=\sum_{F \in \mathrm{SF}^{\bullet}(G)} a_{n}^{|E F|} b_{n}^{|E G|-|E F|}=\sum_{m}\left|\mathrm{SF}_{m}^{\bullet}(G)\right| a_{n}^{m} b_{n}^{s-m}, \\
& b_{n+1}=\sum_{F \in \mathrm{SF}^{\circ}(G)} a_{n}^{|E F|} b_{n}^{|E G|-|E F|}=\sum_{m}\left|\mathrm{SF}_{m}^{\circ}(G)\right| a_{n}^{m} b_{n}^{s-m} .
\end{aligned}
$$

Using the polynomials $p, q, r$ given by

$$
\begin{gathered}
p(x)=x^{d} q(x)=\sum_{m=d}^{\delta}\left|\mathrm{SF}_{m}^{\bullet}(G)\right| x^{m}=\left|\mathrm{SF}_{d}^{\bullet}(G)\right| x^{d}+\cdots+\left|\mathrm{SF}_{\delta}^{\bullet}(G)\right| x^{\delta} \\
r(x)=\sum_{m=0}^{\delta-1}\left|\mathrm{SF}_{m}^{\circ}(G)\right| x^{m}=1+\cdots+\left|\mathrm{SF}_{\delta-1}^{\circ}(G)\right| x^{\delta-1}
\end{gathered}
$$


the previous recursion can be reformulated as

$$
a_{n+1}=b_{n}^{s} p\left(x_{n}\right)=b_{n}^{s} x_{n}^{d} q\left(x_{n}\right) \quad \text { and } \quad b_{n+1}=b_{n}^{s} r\left(x_{n}\right),
$$

yielding a recursion for the quotient $x_{n}=\frac{a_{n}}{b_{n}}$ :

$$
x_{n+1}=f\left(x_{n}\right)=x_{n}^{d} g\left(x_{n}\right) .
$$

Here the rational functions $f$ and $g$ are given by

$$
f(x)=\frac{p(x)}{r(x)} \quad \text { and } \quad g(x)=\frac{q(x)}{r(x)}
$$

respectively. The degrees of $p$ and $r$ are given by $\operatorname{deg}(p)=\delta$ and $\operatorname{deg}(r)=\delta-1$ (recall that $\delta=|V G|-1$ ). It is easy to see that $\mathrm{SF}_{\delta}^{\bullet}(G)$ is the set of spanning trees in $G$ and $\mathrm{SF}_{\delta-1}^{\circ}(G)$ is the set of spanning forests in $G$ with two components each of which contains exactly one distinguished vertex. In Bollobas's book [2] such spanning forests are called thickets. Note that asymptotic information about the number of spanning forests in $X_{n}$ is closely related to the dynamical behavior of $f$. This idea was already used in the work [3].

Lemma 3. Let us note two basic inequalities for $\left|\mathrm{SF}_{m-1}^{\circ}(G)\right|$ and $\left|\mathrm{SF}_{m}^{\bullet}(G)\right|$ :

- We have $\left|\mathrm{SF}_{m-1}^{\circ}(G)\right| \geq \rho\left|\mathrm{SF}_{m}^{\bullet}(G)\right|$.

- Assume that $d=d_{G}(v, w)=1$, then $\left|\mathrm{SF}_{m-1}^{\circ}(G)\right| \leq\left|\mathrm{SF}_{m}^{\bullet}(G)\right|$.

Proof. In order to prove the first part of the lemma, we introduce $\mathrm{SF}^{\otimes}(G)$ to be the set of all spanning forests in $\mathrm{SF}^{\circ}(G)$, where $v$ and $w$ can be connected by one additional edge, and let $B \subseteq V G$ be a vertex subset with $v, w \in B$, so that the induced subgraph $G[B]$ is connected. Then there is a relation between the number $\tau(G[B])$ of spanning trees in $G[B]$ and the number $\theta(G[B])$ of thickets in $G[B]$ with respect to $v, w$ :

$$
\theta(G[B])=\rho(G[B]) \tau(G[B]),
$$

where $\rho(G[B])$ is the resistance scaling factor of $G[B]$ with respect to $v, w$, see [15]; in fact this formula is a consequence of Kirchhoff's famous theorem on electrical currents and spanning trees, see [9] or [2]. Because of Rayleigh's Monotonicity Law for electrical networks (see for example [7]) we have $\rho(G[B]) \geq$ $\rho(G)=\rho$. Now define $\mathrm{SF}^{\otimes}(G, B)$ to be the set of all forests in $\mathrm{SF}^{\otimes}(G)$, where the components of $v$ and $w$ have union $B$, and define $\mathrm{SF}^{\bullet}(G, B)$ to be the set of forests in $\mathrm{SF}^{\bullet}(G)$, where the component of $v$ and $w$ is $B$. Since $\mathrm{SF}^{\otimes}(G, B)$ and $\mathrm{SF}^{\bullet}(G, B)$ only impose restrictions with respect to $v$ and $w$ within the set $B$, it follows that

holds. A simple decomposition yields

$$
\frac{\left|\mathrm{SF}_{m-1}^{\otimes}(G, B)\right|}{\left|\mathrm{SF}_{m}^{\bullet}(G, B)\right|}=\frac{\theta(G[B])}{\tau(G[B])}=\rho(G[B]) \geq \rho(G)=\rho
$$

$$
\left|\mathrm{SF}_{m-1}^{\circ}(G)\right| \geq\left|\mathrm{SF}_{m-1}^{\otimes}(G)\right|=\sum_{B}\left|\mathrm{SF}_{m-1}^{\otimes}(G, B)\right| \geq \sum_{B} \rho\left|\mathrm{SF}_{m}^{\bullet}(G, B)\right|=\rho\left|\mathrm{SF}_{m}^{\bullet}(G)\right|,
$$

where the sums are taken over all vertex subsets $B \subseteq V G$ with $v, w \in B$, so that the induced graph $G[B]$ is connected.

For the second statement of the lemma, note that a spanning forest in $\mathrm{SF}_{m-1}^{\circ}(G)$ must not contain an edge connecting $v$ and $w$. Adding such an edge to a spanning forest in $\operatorname{SF}_{m-1}^{\circ}(G)$ yields a forest in $\mathrm{SF}_{m}^{\bullet}(G)$, proving the inequality. 
Proposition 4. The asymptotic behavior of $f$ at 0 is given by $f(x)=O\left(x^{d}\right)$, whereas the expansion at $\infty$ is given by $f(x)=\rho^{-1} x-c+O\left(x^{-1}\right)$. Here $c \geq 0$ is some constant, which is positive, unless all simple paths (i.e. paths which do not visit vertices several times) connecting $v$ and $w$ have length 1 . In this case the function $f$ is given by $f(x)=\rho^{-1} x$.

Proof. Both asymptotic expansions follow from the definitions of $f$. The constant $c$ is given by

$$
c=\frac{\left|\mathrm{SF}_{\delta}^{\bullet}(G)\right| \cdot\left|\mathrm{SF}_{\delta-2}^{\circ}(G)\right|-\left|\mathrm{SF}_{\delta-1}^{\bullet}(G)\right| \cdot\left|\mathrm{SF}_{\delta-1}^{\circ}(G)\right|}{\left|\mathrm{SF}_{\delta-1}^{\circ}(G)\right|^{2}}
$$

This constant is positive if and only if

$$
\rho=\frac{\left|\mathrm{SF}_{\delta-1}^{\circ}(G)\right|}{\left|\mathrm{SF}_{\delta}^{\bullet}(G)\right|}<\frac{\left|\mathrm{SF}_{\delta-2}^{\circ}(G)\right|}{\left|\mathrm{SF}_{\delta-1}^{\bullet}(G)\right|}
$$

A close inspection of the proof of Lemma 3 shows that $\left|\mathrm{SF}_{\delta-2}^{\circ}(G)\right|>\rho\left|\mathrm{SF}_{\delta-1}^{\bullet}(G)\right|$ holds, unless all simple paths between $v$ and $w$ have length 1 .

The last part of the statement follows from the fact that

$$
\left|\mathrm{SF}_{m-1}^{\circ}(G)\right|=\rho\left|\mathrm{SF}_{m}^{\bullet}(G)\right|
$$

holds, since every forest in $\mathrm{SF}_{m-1}^{\circ}(G)$ corresponds to exactly $\rho^{-1} \in \mathbb{N}$ forests in $\mathrm{SF}_{m}^{\bullet}(G)$ in this case.

Corollary 5. The function $f$ satisfies $f(x) \leq \rho^{-1} x$ and thus $x_{n} \leq \rho^{-n} x_{0}$. If not all simple paths connecting $v$ and $w$ have length 1 , then $f(x)<\rho^{-1} x$ for $x>0$.

If there is an edge connecting $v$ and $w$, then $f(x) \geq x$. Furthermore, if this edge is not a bridge, then $f(x)>x$ for $x>0$, whence $x_{n} \rightarrow \infty$ for $n \rightarrow \infty$.

Proof. The first statement follows easily from Lemma 3

$$
x r(x)=\sum_{m}\left|\operatorname{SF}_{m-1}^{\circ}(G)\right| x^{m} \geq \rho \sum_{m}\left|\operatorname{SF}_{m}^{\bullet}(G)\right| x^{m}=\rho p(x)
$$

The improved inequality follows from the proof of Lemma 3

In order to prove the second part, assume that $v$ and $w$ are connected by an edge. Then a similar computation as before yields $f(x) \geq x$. If the edge connecting $v$ and $w$ is not a bridge, then $\left|\mathrm{SF}_{m-1}^{\circ}(G)\right|<\left|\mathrm{SF}_{m}^{\bullet}(G)\right|$ for some $m$. Hence $f(x)>x$ for $x>0$ in this case.

Lemma 6. Assume that $x_{n}$ is bounded from above. Then there is a constant $\beta_{1}>1$, so that $b_{n}=$ $\beta_{1}^{s^{n}+O(1)}$. If $x_{n}$ converges, then the error term can be improved:

$$
b_{n}=C_{1} \beta_{1}^{s^{n}}(1+o(1))
$$

for some constant $C_{1}>0$. 
Proof. Using Equation (1) we obtain

$$
\log b_{n}=s \log b_{n-1}+\log r\left(x_{n-1}\right)=s^{n}\left(\log b_{0}+\sum_{k=0}^{n-1} s^{-k-1} \log r\left(x_{k}\right)\right)
$$

by iteration. Define the constant $K_{1}$ by

$$
K_{1}=\log b_{0}+\sum_{k=0}^{\infty} s^{-k-1} \log r\left(x_{k}\right)
$$

(the sum converges due to the boundedness of $x_{n}$ and $r(x) \geq 1$ for $x \geq 0$ ). This further implies

$$
\log b_{n}=K_{1} s^{n}+O(1) \quad \text { and } \quad b_{n}=\beta_{1}^{s^{n}+O(1)},
$$

where $\beta_{1}=\exp \left(K_{1}\right)$. Suppose that $x_{n} \rightarrow x_{\infty}$ holds, then the second part follows from

$$
\begin{aligned}
\log b_{n} & =s^{n}\left(K_{1}-\sum_{k=n}^{\infty} s^{-k-1} \log r\left(x_{k}\right)\right) \\
& =s^{n}\left(K_{1}-\sum_{k=n}^{\infty} s^{-k-1} \log r\left(x_{\infty}\right)+O\left(\sum_{k=n}^{\infty} s^{-k-1}\left(x_{k}-x_{\infty}\right)\right)\right) \\
& =s^{n}\left(K_{1}-\frac{s^{-n} \log r\left(x_{\infty}\right)}{s-1}+o\left(s^{-n}\right)\right)=K_{1} s^{n}-\frac{1}{s-1} \log r\left(x_{\infty}\right)+o(1) .
\end{aligned}
$$

Lemma 7. Assume that $x_{n}$ is bounded from above and that $d>1$. Then there is a constant $\beta_{2} \geq 1$, so that $x_{n}=\beta_{2}^{-d^{n}+O(1)}$. If $x_{n}$ converges, then the error term can be improved:

$$
x_{n}=C_{x} \beta_{2}^{-d^{n}}(1+o(1))
$$

for some constant $C_{x}>0$.

Proof. Since the sequence $x_{n}$ is bounded from above, there is a constant $c$ so that $x_{n} \in[0, c]$. For convenience set $y_{n}=\frac{b_{n}}{a_{n}}$. Then $y_{n+1}=y_{n}^{d} g\left(x_{n}\right)^{-1}$ and

$$
\log y_{n}=d \log y_{n-1}-\log g\left(x_{n-1}\right)=d^{n}\left(\log y_{0}-\sum_{k=0}^{n-1} d^{-k-1} \log g\left(x_{k}\right)\right) .
$$

Now define $K_{2}$ by

$$
K_{2}=\log y_{0}-\sum_{k=0}^{\infty} d^{-k-1} \log g\left(x_{k}\right)
$$

The sum involved in the definition of $K_{2}$ converges, since $x_{n} \in[0, c]$ and $g$ is bounded on $[0, c]$ by positive constants. This yields

$$
\log y_{n}=K_{2} d^{n}+O(1) \quad \text { and } \quad x_{n}=\beta_{2}^{-d^{n}+O(1)},
$$


where $\beta_{2}=\exp \left(K_{2}\right)$. Since $x_{n}$ is bounded, it follows that $\beta_{2} \geq 1$. The improvement of the error term is obtained in the same way as in the proof of Lemma 6 .

$$
\log y_{n}=K_{2} d^{n}+\frac{1}{d-1} \log g\left(x_{\infty}\right)+o(1)
$$

where $x_{\infty}$ denotes the limit of $x_{n}$.

Lemma 8. Assume that $\rho<1$ and that $x_{n}$ is not bounded from above, then there are constants $\beta_{3}>$ $1, C_{3}>0, C_{4}>0$, so that

$$
\begin{aligned}
& a_{n}=\left|\mathrm{SF}^{\bullet}\left(X_{n}\right)\right|=C_{3} \rho^{-\frac{s-\delta}{s-1} n} \beta_{3}^{s^{n}}\left(1+O\left(\rho^{n}\right)\right), \\
& b_{n}=\left|\mathrm{SF}^{\circ}\left(X_{n}\right)\right|=C_{4} \rho^{\frac{\delta-1}{s-1} n} \beta_{3}^{s^{n}}\left(1+O\left(\rho^{n}\right)\right) .
\end{aligned}
$$

Proof. Using Proposition 4, it is easy to see that $x_{n}$ tends exponentially to $\infty$. Furthermore, it follows from Equation (1), that the quotient $y_{n}=\frac{b_{n}}{a_{n}}$ satisfies

$$
y_{n+1}=y_{n} \frac{\tilde{r}\left(y_{n}\right)}{\tilde{q}\left(y_{n}\right)}
$$

where $\tilde{q}$ and $\tilde{r}$ are the reversed polynomials of $q$ and $r$, respectively. Since

$$
\frac{\tilde{r}(0)}{\tilde{q}(0)}=\frac{\left|\mathrm{SF}_{\delta-1}^{\circ}(G)\right|}{\left|\mathrm{SF}_{\delta}^{\circ}(G)\right|}=\rho
$$

the product

$$
P=\prod_{k=0}^{\infty} \frac{\tilde{r}\left(y_{k}\right)}{\rho \tilde{q}\left(y_{k}\right)}
$$

converges due to the exponential decay of $y_{n}$. This implies that $y_{n}=P \rho^{n} y_{0}\left(1+\varepsilon_{n}\right)$, where $\varepsilon_{n}=O\left(\rho^{n}\right)$. Using Equation (1) we get $a_{n+1}=a_{n}^{s} y_{n}^{s-\delta} \tilde{q}\left(y_{n}\right)$. Define $\varepsilon_{n}^{\prime}$ by the equation

$$
\tilde{q}\left(y_{n}\right)\left(1+\varepsilon_{n}\right)^{s-\delta}=\left|\mathrm{SF}_{\delta}^{\bullet}(G)\right|\left(1+\varepsilon_{n}^{\prime}\right)
$$

Note that $\varepsilon_{n}^{\prime}=O\left(\rho^{n}\right)$. Altogether we obtain $a_{n+1}=a_{n}^{s} \rho^{n(s-\delta)} C\left(1+\varepsilon_{n}^{\prime}\right)$, where the constant $C$ is given by $C=\left(P y_{0}\right)^{s-\delta}\left|\mathrm{SF}_{\delta}^{\bullet}(G)\right|$. This implies

$$
\log a_{n}=s^{n}\left(\log a_{0}+\sum_{k=0}^{n-1} s^{-k-1}\left(k(s-\delta) \log \rho+\log C+\log \left(1+\varepsilon_{k}^{\prime}\right)\right)\right) .
$$

Now define the constant $K_{3}$ by the sum

$$
K_{3}=\log a_{0}+\sum_{k=0}^{\infty} s^{-k-1}\left(k(s-\delta) \log \rho+\log C+\log \left(1+\varepsilon_{k}^{\prime}\right)\right) .
$$


It follows that

$$
\begin{aligned}
\log a_{n} & =s^{n}\left(K_{3}-\sum_{k=n}^{\infty} s^{-k-1}(k(s-\delta) \log \rho+\log C)-\sum_{k=n}^{\infty} s^{-k-1} \log \left(1+\varepsilon_{k}^{\prime}\right)\right) \\
& =K_{3} s^{n}-\frac{n(s-\delta) \log \rho}{s-1}-\frac{\log C}{s-1}-\frac{(s-\delta) \log \rho}{(s-1)^{2}}+O\left(\rho^{n}\right)
\end{aligned}
$$

and

$$
\begin{aligned}
\log b_{n} & =\log a_{n}+\log y_{n} \\
& =K_{3} s^{n}+\frac{n(\delta-1) \log \rho}{s-1}+\log \left(P y_{0}\right)-\frac{\log C}{s-1}-\frac{(s-\delta) \log \rho}{(s-1)^{2}}+O\left(\rho^{n}\right)
\end{aligned}
$$

proving the statement.

Now we are prepared to prove Theorem 1

Proof of Theorem 1 We distinguish several cases:

(A) Assume that $\rho \geq 1$. Then $x_{n} \leq \rho^{-n} x_{0}$ by Corollary 5

- $\rho>1, d>1$ : Since $\rho>1$, the sequence $x_{n}$ decays exponentially to 0 . Hence Lemma 6 and Lemma 7 imply asymptotic expansions of $a_{n}$ and $b_{n}$ with improved error terms. Note that $r(0)=1$ and $q(0)=\left|\mathrm{SF}_{d}^{\bullet}(G)\right| \geq 1$.

- $\rho=1, d>1$ : Corollary 5 yields $f(x)<x$ for $x>0$. Hence the sequence $x_{n}$ tends to 0 and an application of Lemma 6 and Lemma 7 shows once again, that the strong version of the asymptotic expansions holds.

- $\rho=1, d=1$ : In this case there is an edge connecting $v$ and $w$, which is a bridge (otherwise $\rho<1$ ). Thus $f(x)=x$ and $b_{n+1}=b_{n}^{s} r\left(x_{0}\right)$, whence it is easy to derive exact formulæ for $a_{n}$ and $b_{n}$.

Since $d=1$ implies $\rho \leq 1$, the case $\rho>1$ and $d=1$ is impossible.

(B) Assume that $\rho<1$ and $d=1$. Then Corollary 5 yields $f(x)>x$ for $x>0$, so that $x_{n}$ tends to $\infty$. In this case Lemma 8 implies the desired asymptotic expansions of $a_{n}$ and $b_{n}$.

(C) Finally, assume that $\rho<1$ and $d>1$. The behavior of $x_{n}$ depends on the initial value $x_{0}$. Due to Proposition 4 both 0 and $\infty$ are attracting fixed points of $f$. Thus we have to distinguish further:

- If $x_{n} \rightarrow 0$, then once again asymptotic expansions of $a_{n}$ and $b_{n}$ in strong forms are obtained from Lemma 6 and Lemma 7

- If $x_{n} \rightarrow \infty$, then asymptotic expansions of $a_{n}$ and $b_{n}$ are implied by Lemma 8

- If $x_{n}$ is not caught by the fixpoints 0 or $\infty$, then $x_{n}$ is bounded by positive constants. This is the only case where we only obtain weak forms of asymptotic expansions for $a_{n}$ and $b_{n}$ by Lemma 6 and Lemma 7 in general. If $x_{n}$ converges in this case, then the error terms improve to the strong forms. 

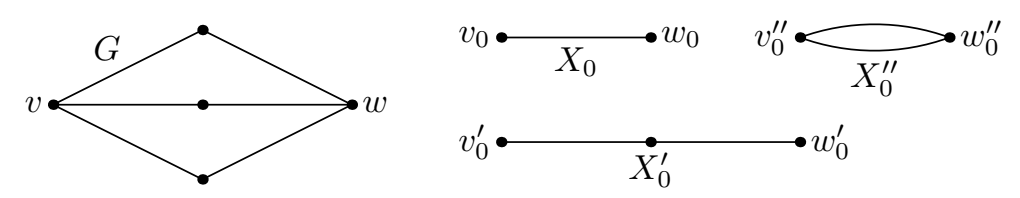

Fig. 4: A model graph $G$ and three initial graphs $X_{0}, X_{0}^{\prime}, X_{0}^{\prime \prime}$.

Example. Consider the model graph $G$ and the initial graphs $X_{0}, X_{0}^{\prime}$ and $X_{0}^{\prime \prime}$ given in Figure 4 The evolution of the sequence $X_{n}$ is shown in Figure 5 . In this case $s=6, d=2, \delta=4$, and $\rho=\frac{2}{3}$. Furthermore, it is easy to compute the polynomials $p$ and $r$ :

$$
p(x)=3 x^{2}+12 x^{3}+12 x^{4} \quad \text { and } \quad r(x)=1+6 x+12 x^{2}+8 x^{3} .
$$

The rational function $f: x \mapsto \frac{p(x)}{r(x)}$ is strictly monotone and convex on $[0, \infty)$. Hence $x=1$ is the only fixpoint in $(0, \infty)$ and this fixpoint is repelling. Finally, it is easy to compute that the ratio $x_{0}$ is given by

$$
x_{0}= \begin{cases}1 & \text { if we take the initial graph } X_{0} \\ \frac{1}{3} & \text { if we take the initial graph } X_{0}^{\prime} \\ 2 & \text { if we take the initial graph } X_{0}^{\prime \prime}\end{cases}
$$

Thus, depending on the initial graph, we obtain different types of asymptotic expansion for the number of spanning forests. Especially, these expansions are given as follows: In the first case

$$
a_{n}=b_{n}=3^{3 / 5\left(6^{n}-1\right)} \approx 0.51728185 \cdot 1.93318204^{6^{n}},
$$

in the second case (note that $x_{\infty}=0$ )

$$
a_{n} \approx \frac{1}{3} \cdot 3.99222335^{6^{n}} \cdot 1.43574175^{-2^{n}} \quad \text { and } \quad b_{n} \approx 3.99222335^{6^{n}}
$$

whereas in the third case we obtain

$$
\begin{aligned}
& a_{n} \approx 0.55221996 \cdot\left(\frac{2}{3}\right)^{-2 n / 5} \cdot 2.67591200^{6^{n}} \\
& b_{n} \approx 0.76288569 \cdot\left(\frac{2}{3}\right)^{3 n / 5} \cdot 2.67591200^{6^{n}} .
\end{aligned}
$$

We conjecture that the function $f$ is always strictly monotone and convex in $[0, \infty)$. As a consequence $f$ has at most one fixpoint in $(0, \infty)$, which is then repelling, so that the sequence $x_{n}$ always converges improving the error term always to $o(1)$.

\section{Connected subgraphs}

Denote by SG $(G)$ the set of all non-empty (not necessarily induced) subgraphs of $G$ and define the following subsets of $\mathrm{SG}(G)$ : For $\nu \in\{0, v, w, 2\}$ let $\mathrm{SG}^{\nu}(G)$ be the set of connected subgraphs in $\mathrm{SG}(G)$ which contain 

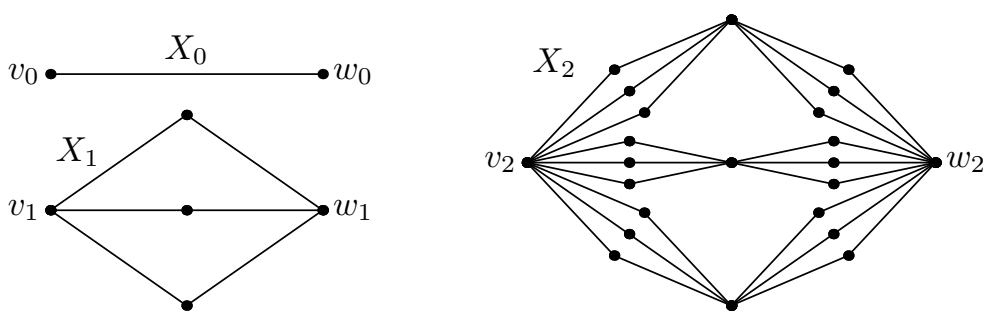

Fig. 5: The graphs $X_{0}, X_{1}, X_{2}$.

- no distinguished vertices, if $\nu=0$,

- the vertex $v$ but not $w$, if $\nu=v$,

- the vertex $w$ but not $v$, if $\nu=w$,

- both distinguished vertices, if $\nu=2$.

Finally let $\mathrm{SG}^{x}(G)$ be the set of all subgraphs with two connected components each of which contains one distinguished vertex. For a subgraph $H$ of $G$ let us define

- $e_{2}(H)$ to be the number of edges in $E H$,

- $e_{v}(H)$ to be the number of edges connecting vertices in $V H$ and $V G \backslash V H$, whose $v$-end lies in $V H$.

- $e_{w}(H)$ to be the number of edges connecting $V H$ and $V G \backslash V H$, whose $w$-end lies in $V H$.

- $e_{x}(H)$ to be the number of edges in $G$ connecting vertices in $V H$ which are not in $E H$.

For the sake of notation set $a_{\nu, n}=\left|\mathrm{SG}^{\nu}\left(X_{n}\right)\right|$ for $\nu \in\{0, v, w, 2, x\}$, where $v$ and $w$ are interpreted as $v_{n}$ and $w_{n}$ in $X_{n}$. Obviously, $a_{\nu, n} \geq 1$ for $\nu \in\{v, w, 2, x\}$ and $n \geq 0$. It is not too difficult to prove that the following system of recursions holds:

$$
a_{\nu, n+1}=[\nu=0] s a_{0, n}+\sum_{H \in \mathrm{SG}^{\nu}(G)} a_{v, n}^{e_{v}(H)} a_{w, n}^{e_{w}(H)} a_{2, n}^{e_{2}(H)} a_{x, n}^{e_{x}(H)} .
$$

Here we have used Iverson notation: $[\nu=0]$ is equal to 1 if $\nu=0$ and 0 otherwise. To see why this holds, simply notice that a connected subgraph on $X_{n+1}$ induces either a connected subgraph, a subgraph of $\mathrm{SG}^{x}$-type, or the empty set on each of the parts that are isomorphic to $X_{n}$. The graphs $H$ describe all possible ways for this. The additional summand in the case $\nu=0$ arises from the possibility that a connected subgraph can be contained in one of the parts (without its boundary vertices) only. Of course, such a connected subgraph cannot contain $v_{n+1}$ or $w_{n+1}$.

The bound $a_{2, n+1} \geq a_{2, n}^{s}$ is a simple consequence of these recursions: Choose $H=G$ in the recursion (2) for $a_{2, n+1}$. Similarly, by choosing appropriate "extremal" subgraphs we obtain

$$
\begin{aligned}
a_{0, n+1} & \geq a_{2, n}^{s-\operatorname{deg}(v)-\operatorname{deg}(w)}, & & a_{v, n+1} \geq a_{2, n}^{s-\operatorname{deg}(w)}, \\
a_{w, n+1} & \geq a_{2, n}^{s-\operatorname{deg}(v)}, & & a_{x, n+1} \geq a_{2, n}^{s-c},
\end{aligned}
$$


where $c$ the number of edges in a minimal $v$ - $w$ cut. As a consequence, $a_{2, n}$ grows at least doubly exponentially if $G$ is not a path. Therefore, for $\nu \in\{0, v, w, x\}$, the quantity $a_{\nu, n}$ also grows at least doubly exponentially in this case.

The proof of Theorem 2 is split into several lemmata. In the following we assume that $v, w$ and $v_{0}, w_{0}$ have degree 1 in $G$ and $X_{0}$, respectively, and write $v^{\prime}$ and $w^{\prime}$ for the unique neighbors of $v$ and $w$ in $G$.

Lemma 9. We have $a_{\nu, n} \geq a_{2, n}$ for $\nu \in\{0, v, w\}$ and

$$
\frac{a_{x, n+1}}{a_{2, n+1}} \geq 2 \cdot \frac{a_{x, n}}{a_{2, n}}
$$

Proof. The first inequality is plain due to the degree restriction: since $v_{n}$ and $w_{n}$ have degree 1 in $X_{n}$, we can obtain a connected subgraph that doesn't contain $w_{n}$ from every connected subgraph that contains $w_{n}$ by removing $w_{n}$ and its incident edge. Hence, $a_{v, n} \geq a_{2, n}$, and likewise for $\nu=w$ and $\nu=0$. In order to prove the second inequality, let us note that every connected subgraph $H$ of $G$ which contains $v$ and $w$ has to contain the edge $v v^{\prime}$ as well as the edge $w w^{\prime}$. Therefore, we can construct two graphs $H_{v}, H_{w} \in \mathrm{SG}^{x}(G)$ from $H$ by removing the edge $v v^{\prime}$ respectively $w w^{\prime}$. Then

$$
e_{\nu}\left(H_{\nu}\right)=e_{\nu}(H), \quad e_{2}\left(H_{\nu}\right)=e_{2}(H)-1, \quad e_{x}\left(H_{\nu}\right)=e_{x}(H)+1
$$

for $\nu \in\{v, w\}$. Together with Equation (2), this shows that

$$
a_{x, n+1} \geq 2 a_{2, n+1} \cdot \frac{a_{x, n}}{a_{2, n}}
$$

proving the statement.

Assume that $G$ is not a path, so that $a_{2,1}>1$. Then we have already noticed that $a_{\nu, n}$ grows at least doubly exponentially for $\nu \in\{0, v, w, 2\}$ if $n \rightarrow \infty$ :

$$
a_{\nu, n} \geq C_{\nu} \alpha^{s^{n}}
$$

for some $\alpha>1$ and constants $C_{\nu}$. Due to this observation it is possible to reduce the recursions given by (2) up to a very small error term: We say that a subgraph $H$ of $G$ is important, if every edge in $G$ has at least one endpoint in $H$, and denote by $\mathrm{SG}_{i}^{\nu}(G)$ the important subgraphs of $\mathrm{SG}^{\nu}(G)$.

Lemma 10. Assume that $G$ is not a path, then for $\nu \in\{0, v, w, 2\}$ the following asymptotic formulae hold:

$$
a_{\nu, n+1}=\left(1+O\left(\alpha^{-s^{n}}\right)\right) \sum_{H \in \mathrm{SG}_{i}^{\nu}(G)} a_{v, n}^{e_{v}(H)} a_{w, n}^{e_{w}(H)} a_{2, n}^{e_{2}(H)} a_{x, n}^{e_{x}(H)}
$$

Proof. Since $a_{\nu, n}$ grows at least doubly exponentially only those summands in the Formulæ (2) can be of asymptotic importance which belong to a subgraph $H$, where $e_{v}(H)+e_{w}(H)+e_{2}(H)+e_{x}(H)$ is maximal. However, this equals $s-e_{r}(H)$, where $e_{r}(H)$ is the number of edges connecting vertices in $V G \backslash V H$. This means that every edge of $G$ must have at least one endpoint in $H$, so that $H$ must be an important subgraph. The error term is then implied by (3).

We note that in Lemma 12 more explicit expressions for $a_{\nu, n+1}$ are derived. 
Lemma 11. Assume that $G$ is not a path. Suppose that $\mathrm{h}_{w}\left(v v^{\prime}\right) \neq v$ or $\mathrm{h}_{v}\left(w w^{\prime}\right) \neq w$, then there exist constants $c_{\nu}$ for $\nu \in\{0, v, w\}$, so that

$$
a_{\nu, n}=c_{\nu} a_{2, n}+O\left(\alpha^{-s^{n}}\right)
$$

holds. If $\mathrm{h}_{w}\left(v v^{\prime}\right)=v$ and $\mathrm{h}_{v}\left(w w^{\prime}\right)=w$, then a distinction on the parity of $n$ is necessary: there are constants $c_{k, \nu}$ for $\nu \in\{0, v, w\}$ and $k \in\{0,1\}$, so that

$$
a_{\nu, n}=c_{k, \nu} a_{2, n}+O\left(\alpha^{-s^{n}}\right)
$$

holds, where $k \in\{0,1\}$ is the remainder of the division of $n$ by 2 .

Proof. Depending on the construction of $X_{n+1}$, there are four possible arrangements with respect to the replacement of the edges $v v^{\prime}$ and $w w^{\prime}$ by copies of $X_{n}$. We deal with two of these four cases, since they are all very similar. Hence assume that $\mathrm{h}_{v}\left(v v^{\prime}\right)=v$ and $\mathrm{h}_{w}\left(w w^{\prime}\right)=w$. Consider an important graph $H$ in $\mathrm{SG}_{i}^{v}(G)$. This graph $H$ must contain $w^{\prime}$ but not $w$. Hence we can construct an important graph $H_{w} \in \mathrm{SG}_{i}^{2}(G)$ by adding the vertex $w$ and the edge $w w^{\prime}$. The mapping $\mathrm{SG}_{i}^{v}(G) \rightarrow \mathrm{SG}_{i}^{2}(G), H \mapsto H_{w}$ is in fact a one-to-one correspondence. Since

$$
\begin{array}{ll}
e_{v}\left(H_{w}\right)=e_{v}(H)-1, & e_{w}\left(H_{w}\right)=e_{w}(H), \\
e_{2}\left(H_{w}\right)=e_{2}(H)+1, & e_{x}\left(H_{w}\right)=e_{x}(H),
\end{array}
$$

it follows that

$$
a_{v, n+1}=a_{2, n+1} \cdot\left(\frac{a_{v, n}}{a_{2, n}}+O\left(\alpha^{-s^{n}}\right)\right)
$$

or

$$
\frac{a_{v, n+1}}{a_{2, n+1}}=\frac{a_{v, n}}{a_{2, n}}+O\left(\alpha^{-s^{n}}\right) .
$$

If $\varepsilon_{n}$ denotes the error term on the right hand side, then

$$
\frac{a_{v, n}}{a_{2, n}}=\frac{a_{v, 0}}{a_{2,0}}+\sum_{k=0}^{n-1} \varepsilon_{k},
$$

which converges to

$$
c_{v}=\frac{a_{v, 0}}{a_{2,0}}+\sum_{k=0}^{\infty} \varepsilon_{k}
$$

and the error term is given by

$$
\frac{a_{v, n}}{a_{2, n}}=c_{v}-\sum_{k=n}^{\infty} \varepsilon_{k}=c_{v}+O\left(\alpha^{-s^{n}}\right) .
$$

Analogous arguments show that

$$
\frac{a_{w, n}}{a_{2, n}}=c_{w}+O\left(\alpha^{-s^{n}}\right)
$$


and

$$
\frac{a_{0, n}}{a_{2, n}}=c_{0}+O\left(\alpha^{-s^{n}}\right)
$$

which implies the statement for this arrangement. If $\mathrm{h}_{v}\left(v v^{\prime}\right)=v, \mathrm{~h}_{w}\left(w w^{\prime}\right)=w^{\prime}$ or $\mathrm{h}_{v}\left(v v^{\prime}\right)=v^{\prime}$, $\mathrm{h}_{w}\left(w w^{\prime}\right)=w$, we obtain the same result by essentially the same method, but if $\mathrm{h}_{v}\left(v v^{\prime}\right)=v^{\prime}$ and $\mathrm{h}_{w}\left(w w^{\prime}\right)=w^{\prime}$, which is equivalent to $\mathrm{h}_{w}\left(v v^{\prime}\right)=v$ and $\mathrm{h}_{v}\left(w w^{\prime}\right)=w$, things are slightly different. Again, there is a one-to-one correspondence $H \mapsto H_{w}$ between $\mathrm{SG}_{i}^{v}(G)$ and $\mathrm{SG}_{i}^{2}(G)$ (constructed as before), but we have

$$
\begin{array}{ll}
e_{v}\left(H_{w}\right)=e_{v}(H), & e_{w}\left(H_{w}\right)=e_{w}(H)-1, \\
e_{2}\left(H_{w}\right)=e_{2}(H)+1, & e_{x}\left(H_{w}\right)=e_{x}(H) .
\end{array}
$$

Therefore, we get

$$
a_{v, n+1}=a_{2, n+1} \cdot\left(\frac{a_{w, n}}{a_{2, n}}+O\left(\alpha^{-s^{n}}\right)\right)
$$

and analogously

$$
a_{w, n+1}=a_{2, n+1} \cdot\left(\frac{a_{v, n}}{a_{2, n}}+O\left(\alpha^{-s^{n}}\right)\right) .
$$

Combining the two, we obtain

$$
a_{v, n+2}=a_{2, n+2} \cdot\left(\frac{a_{v, n}}{a_{2, n}}+O\left(\alpha^{-s^{n}}\right)\right),
$$

and following the above arguments we find that $a_{v, n}$ converges to some value $c_{0, v}$ for even $n$ and some value $c_{1, v}$ for odd $n$, which makes the distinction of the two cases necessary. The rest of the proof is analogous to the first case again.

As before we write $\tau(G)$ for the number of spanning trees in $G$ and $\theta(G)$ for the number of thickets in $G$ with respect to $v$ and $w$. Furthermore, recall that $\delta=|V G|-1$ is the number of edges in a spanning tree of $G$. A vertex of degree 1 is called a leaf and we write $\ell_{v}$ to denote the number of leaves (except $v, w)$ of $G$, which are not $v$-ends. Let $t_{v}$ be equal to $v$, if $w$ is the $w$-end of the edge $w w^{\prime}$, or equal to $w$ otherwise. We use $\ell_{w}$ and $t_{w}$ analogous to $\ell_{v}$ and $t_{v}$, respectively. For simplicity let $\ell=\ell_{v}+\ell_{w}$ be the number of leaves (except $v, w)$ in $G$.

Lemma 12. If $G$ is not a path, then, for $\nu \in\{v, w\}$,

$$
\begin{aligned}
& a_{0, n+1}=\tau(G) a_{t_{v}, n} a_{t_{w}, n} a_{2, n}^{\delta-\ell-2}\left(a_{v, n}+a_{2, n}\right)^{\ell_{v}}\left(a_{w, n}+a_{2, n}\right)^{\ell_{w}} a_{x, n}^{s-\delta}\left(1+O\left(2^{-n}\right)\right), \\
& a_{\nu, n+1}=\tau(G) a_{t_{\nu}, n} a_{2, n}^{\delta-\ell-1}\left(a_{v, n}+a_{2, n}\right)^{\ell_{v}}\left(a_{w, n}+a_{2, n}\right)^{\ell_{w}} a_{x, n}^{s-\delta}\left(1+O\left(2^{-n}\right)\right), \\
& a_{2, n+1}=\tau(G) a_{2, n}^{\delta-\ell}\left(a_{v, n}+a_{2, n}\right)^{\ell_{v}}\left(a_{w, n}+a_{2, n}\right)^{\ell_{w}} a_{x, n}^{s-\delta}\left(1+O\left(2^{-n}\right)\right), \\
& a_{x, n+1}=\theta(G) a_{2, n}^{\delta-\ell-1}\left(a_{v, n}+a_{2, n}\right)^{\ell_{v}}\left(a_{w, n}+a_{2, n}\right)^{\ell_{w}} a_{x, n}^{s-\delta+1}\left(1+O\left(2^{-n}\right)\right) .
\end{aligned}
$$

Proof. By Lemma 11 the quantities $a_{v, n}$ for $\nu \in\{0, v, w, 2\}$ are of the same asymptotic order. Lemma 9 implies $a_{x, n} \geq c 2^{n} a_{2, n}$ for some constant $c$. Consequently, only those summands in the Equations (2) are of interest which belong to an important subgraph $H$, where $e_{x}(H)$ is maximal (all the other summands 
are smaller by an exponential factor). Certainly, $H$ must not contain any circles, since we could remove edges in this case to increase $e_{x}(H)$ and decrease $e_{2}(H)$ instead. Furthermore, $V G \backslash V H$ contains leaves only. Otherwise, assume that there is a vertex $u$ in $V G \backslash V H$ that is not a leaf. Since the subgraph $H$ is important, all neighbors of this vertex have to be in $V H$, and there are at least two of them, since $u$ is not a leaf. Now we can add $u$ and an edge between $u$ and one of its neighbors to $H$ to obtain a new important subgraph $H^{\prime}$, and $e_{x}\left(H^{\prime}\right)>e_{x}(H)$ (there is at least one edge between $u$ and one of the vertices in $V H^{\prime}$ that doesn't belong to $E H^{\prime}$ and therefore contributes to $e_{x}\left(H^{\prime}\right)$, but not to $e_{x}(H)$ ).

Now consider for instance the recurrence for $a_{2, n+1}$ in (2). Each important subgraph $H$ in the sum for which $e_{x}(H)$ is maximized is obtained by taking a spanning tree and possibly removing some of the leaves (since it must be connected and cycle-free and contain all vertices except possibly for the leaves). There are $\tau(G)$ choices for the spanning tree, and for each of the leaves we may choose whether to include it or not. Edges whose one endpoint is a leaf contribute a factor $a_{v, n}$ (if the leaf is not included and a $w$ end), a factor $a_{w, n}$ (if the leaf is not included and a $v$-end) or a factor $a_{2, n}$ (if the leaf is included). The remaining edges contribute a factor of $a_{2, n}$ if they are contained in $H$ (there are always precisely $\delta-\ell$ such edges) and $a_{x, n}$ otherwise (there are precisely $s-\delta$ such edges). Combining everything, we obtain the equation for $a_{2, n+1}$. The other three equations follow analogously.

Corollary 13. If $G$ is not a path, then there is a constant $c_{x}$, so that

$$
a_{x, n}=c_{x} \rho^{n} a_{2, n}\left(1+O\left(2^{-n}\right)\right) .
$$

Proof. Divide the last two equations in the previous lemma to find

$$
\frac{a_{x, n+1}}{a_{2, n+1}}=\frac{\theta(G)}{\tau(G)} \cdot \frac{a_{x, n}}{a_{2, n}}\left(1+O\left(2^{-n}\right)\right)=\rho \cdot \frac{a_{x, n}}{a_{2, n}}\left(1+O\left(2^{-n}\right)\right) .
$$

Now we can apply the same technique as in Lemma 8 to show that

$$
\frac{a_{x, n}}{a_{2, n}}=c_{x} \rho^{n}\left(1+O\left(2^{-n}\right)\right)
$$

for some constant $c_{x}$, which concludes the proof of this lemma.

Proof of Theorem 2 Suppose that $\mathrm{h}_{w}\left(v v^{\prime}\right) \neq v$ or $\mathrm{h}_{v}\left(w w^{\prime}\right) \neq w$. Using Lemma 11, Lemma 12, and Corollary 13 we obtain

$$
a_{2, n+1}=C \rho^{n(s-\delta)} a_{2, n}^{s}\left(1+O\left(2^{-n}\right)\right)
$$

for some constant $C$. The methods employed in the proof of Lemma 8 now yield the statement. The case $\mathrm{h}_{w}\left(v v^{\prime}\right)=v$ and $\mathrm{h}_{v}\left(w w^{\prime}\right)=w$ is similar.

We conjecture that a result similar to Theorem 2 holds, when the assumption that $v, w$ are leaves is replaced by $\rho>1$. The following example indicates the differences and problems. 


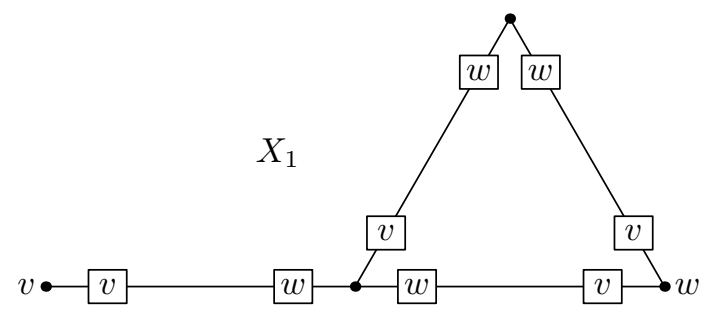

Fig. 6: The first stage in the sequence of Austria graphs. Boxed labels indicate $v$-ends and $w$-ends of edges.

Example. Let us consider the number of connected subgraphs in the sequence of Austria graphs, see Example 2. Using Formula (2) and Figure 6 we obtain:

$$
\begin{aligned}
& a_{0, n+1}=4 a_{0, n}+a_{w, n}^{2}+a_{v, n} a_{w, n}^{2}+a_{w, n}^{3} a_{2, n} \\
& a_{v, n+1}=a_{v, n}+a_{v, n} a_{w, n} a_{2, n}+a_{w, n}^{2} a_{2, n}^{2} \\
& a_{w, n+1}=a_{v, n}^{2}+a_{v, n} a_{w, n} a_{2, n}+a_{v, n}^{2} a_{w, n} a_{2, n}+a_{w, n} a_{2, n}^{3}+3 a_{w, n} a_{2, n}^{2} a_{x, n} \\
& a_{2, n+1}=a_{v, n}^{2} a_{2, n}^{2}+a_{2, n}^{4}+3 a_{2, n}^{3} a_{x, n} \\
& a_{x, n+1}=a_{v, n}^{3}+a_{v, n}^{2} a_{w, n} a_{2, n}+2 a_{v, n}^{2} a_{2, n} a_{x, n}+a_{2, n}^{3} a_{x, n}+5 a_{2, n}^{2} a_{x, n}^{2}
\end{aligned}
$$

Since all the numbers $a_{\nu, n}$ grow at least doubly exponentially, say $a_{\nu, n} \geq C_{\nu} \zeta^{4^{n}}$ (for some constants $C_{\nu}$ and $\zeta>1$ ), the terms of total degree 4 are much larger than the others. So we have to study the following:

$$
\begin{aligned}
& a_{0, n+1}=a_{w, n}^{3} a_{2, n}\left(1+O\left(\zeta^{-4^{n}}\right)\right), \\
& a_{v, n+1}=a_{w, n}^{2} a_{2, n}^{2}\left(1+O\left(\zeta^{-4^{n}}\right)\right), \\
& a_{w, n+1}=a_{w, n}\left(a_{v, n}^{2} a_{2, n}+a_{2, n}^{3}+3 a_{2, n}^{2} a_{x, n}\right)\left(1+O\left(\zeta^{-4^{n}}\right)\right), \\
& a_{2, n+1}=a_{2, n}\left(a_{v, n}^{2} a_{2, n}+a_{2, n}^{3}+3 a_{2, n}^{2} a_{x, n}\right), \\
& a_{x, n+1}=\left(a_{v, n}^{2} a_{w, n} a_{2, n}+2 a_{v, n}^{2} a_{2, n} a_{x, n}+a_{2, n}^{3} a_{x, n}+5 a_{2, n}^{2} a_{x, n}^{2}\right)\left(1+O\left(\zeta^{-4^{n}}\right)\right) .
\end{aligned}
$$

It follows that

$$
\frac{a_{0, n+1}}{a_{v, n+1}}=\frac{a_{w, n}}{a_{2, n}} \cdot\left(1+O\left(\zeta^{-4^{n}}\right)\right), \quad \frac{a_{w, n+1}}{a_{2, n+1}}=\frac{a_{w, n}}{a_{2, n}} \cdot\left(1+O\left(\zeta^{-4^{n}}\right)\right),
$$

so that

$$
a_{0, n}=c a_{v, n}\left(1+O\left(\zeta^{-4^{n}}\right)\right) \quad \text { and } \quad a_{w, n}=c a_{2, n}\left(1+O\left(\zeta^{-4^{n}}\right)\right)
$$

for some constant $c$. By removing the edge incident to $v_{n}$ from a connected subgraph in $\mathrm{SG}^{2}\left(X_{n}\right)$ a subgraph in $S G^{x}\left(X_{n}\right)$ is obtained. Thus $a_{x, n} \geq a_{2, n}$. Furthermore, using the recursions we get

$$
\frac{a_{x, n+1}}{a_{2, n+1}} \geq \frac{2 a_{v, n}^{2} a_{2, n} a_{x, n}+a_{2, n}^{3} a_{x, n}+5 a_{2, n}^{2} a_{x, n}^{2}}{a_{v, n}^{2} a_{2, n}^{2}+a_{2, n}^{4}+3 a_{2, n}^{3} a_{x, n}}=\frac{a_{x, n}}{a_{2, n}} \cdot\left(1+\frac{a_{v, n}^{2}+2 a_{2, n} a_{x, n}}{a_{v, n}^{2}+a_{2, n}^{2}+3 a_{2, n} a_{x, n}}\right) .
$$

Since

$$
2\left(a_{v, n}^{2}+2 a_{2, n} a_{x, n}\right) \geq 2 a_{v, n}^{2}+a_{2, n}^{2}+3 a_{2, n} a_{x, n} \geq a_{v, n}^{2}+a_{2, n}^{2}+3 a_{2, n} a_{x, n}
$$


the inequality

$$
\frac{a_{x, n+1}}{a_{2, n+1}} \geq \frac{3}{2} \cdot \frac{a_{x, n}}{a_{2, n}}
$$

follows. Using this estimate we obtain

$$
a_{x, n} \geq c^{\prime}\left(\frac{3}{2}\right)^{n} a_{2, n} \quad \text { and } \quad a_{w, n} \geq c^{\prime \prime}\left(\frac{3}{2}\right)^{n} a_{v, n},
$$

for some constants $c^{\prime}$ and $c^{\prime \prime}$, since

$$
\frac{a_{w, n+1}}{a_{v, n+1}} \geq \frac{3 a_{w, n} a_{2, n}^{2} a_{x, n}}{a_{w, n}^{2} a_{2, n}^{2}} \cdot\left(1+O\left(\zeta^{-4^{n}}\right)\right)=\frac{3 a_{x, n}}{a_{w, n}} \cdot\left(1+O\left(\zeta^{-4^{n}}\right)\right) .
$$

As a consequence we can further simplify the recursions:

$$
\begin{aligned}
a_{0, n+1} & =a_{w, n}^{3} a_{2, n}\left(1+O\left(\zeta^{-4^{n}}\right)\right), \\
a_{v, n+1} & =a_{w, n}^{2} a_{2, n}^{2}\left(1+O\left(\zeta^{-4^{n}}\right)\right), \\
a_{w, n+1} & =3 a_{w, n} a_{2, n}^{2} a_{x, n}\left(1+O\left(\left(\frac{2}{3}\right)^{n}\right)\right), \\
a_{2, n+1} & =3 a_{2, n}^{3} a_{x, n}\left(1+O\left(\left(\frac{2}{3}\right)^{n}\right)\right), \\
a_{x, n+1} & =5 a_{2, n}^{2} a_{x, n}^{2}\left(1+O\left(\left(\frac{2}{3}\right)^{n}\right)\right) .
\end{aligned}
$$

Using the last two relations we get

$$
a_{x, n}=c_{x}\left(\frac{5}{3}\right)^{n} a_{2, n}\left(1+O\left(\left(\frac{2}{3}\right)^{n}\right)\right)
$$

for some $c_{x}$. Now the methods of Lemma 8 yield

$$
a_{\nu, n}=C_{\nu}\left(\frac{5}{3}\right)^{\left(2 / 3-k_{\nu}\right) n} \beta^{4^{n}}\left(1+O\left(\left(\frac{2}{3}\right)^{n}\right),\right.
$$

where

$$
k_{\nu}= \begin{cases}0 & \text { if } \nu=x \\ 1 & \text { if } \nu \in\{2, w\}, \\ 2 & \text { if } \nu \in\{v, 0\} .\end{cases}
$$

The approximate numerical value of $\beta$ is 1.77280776 , whereas $\left(C_{0}, C_{v}, C_{w}, C_{2}, C_{x}\right)$ is approximately equal to

$$
\text { (1.13215234, 0.793383233, 0.950775521, 0.666279023, 0.632751624). }
$$

For comparison with Theorem 2 we recall that $\rho=\frac{5}{3}$ and that the exponent of $\rho$ in this theorem is given by

$$
-\frac{s-\delta}{s-1}=-\frac{1}{3}
$$

Although the number of connected subgraphs is given by the same asymptotic form as before, the quantities $a_{\nu, n}$ show a different behavior. 
We notice that the asymptotics derived in the previous example always holds, when $\operatorname{deg}\left(v_{n}\right)=1$ and $\operatorname{deg}\left(w_{n}\right)=c>1$ or vice versa. The argument for this follows the line of the proof of Theorem 2 with suitable modifications. The final example demonstrates that quite a different asymptotic behavior is possible if the resistance scaling factor $\rho$ is less than 1 .

Example. We consider the graphs $X_{n}$ defined in Example 3. Since the graphs $X_{n}$ are symmetric with respect to $v_{n}$ and $w_{n}$, the quantities $a_{v, n}$ and $a_{w, n}$ are the same: Thus set $a_{1, n}=a_{v, n}=a_{w, n}$. Then we get

$$
\begin{aligned}
& a_{0, n+1}=6 a_{0, n}+3 a_{1, n}^{2} \\
& a_{1, n+1}=a_{1, n}^{3}+3 a_{1, n}^{3} a_{2, n}+3 a_{1, n}^{3} a_{2, n}^{2}+a_{1, n}^{3} a_{2, n}^{3}=a_{1, n}^{3}\left(1+a_{2, n}\right)^{3} \\
& a_{2, n+1}=3 a_{1, n}^{4} a_{2, n}^{2}+3 a_{1, n}^{2} a_{2, n}^{4}+a_{2, n}^{6}+12 a_{1, n}^{2} a_{2, n}^{3} a_{x, n}+12 a_{2, n}^{4} a_{x, n}^{2}+6 a_{2, n}^{5} a_{x, n} \\
& a_{x, n+1}=a_{1, n}^{6}+6 a_{1, n}^{4} a_{2, n} a_{x, n}+12 a_{1, n}^{2} a_{2, n}^{2} a_{x, n}^{2}+8 a_{2, n}^{3} a_{x, n}^{3}=\left(a_{1, n}^{2}+2 a_{2, n} a_{x, n}\right)^{3} .
\end{aligned}
$$

As usual the numbers $a_{\nu, n}(\nu \in\{0,1,2, x\})$ grow at least doubly exponentially, say $a_{\nu, n} \geq c_{\nu} \zeta^{6^{n}}$, it follows that

$$
\frac{a_{2, n+1}}{a_{1, n+1}} \geq \frac{3 a_{1, n}^{2} a_{2, n}^{4}}{a_{1, n}^{3}\left(1+a_{2, n}\right)^{3}} \geq 3 \cdot \frac{a_{2, n}}{a_{1, n}} \cdot\left(1+O\left(\zeta^{-6^{n}}\right)\right)
$$

We have $a_{1, n} \leq a_{x, n}$ by induction: $a_{1, n+1} \leq 8 a_{1, n}^{3} a_{2, n}^{3} \leq 8 a_{2, n}^{3} a_{x, n}^{3} \leq a_{x, n+1}$. As a consequence it follows that

$$
\begin{aligned}
\frac{a_{x, n+1}}{a_{1, n+1}} & =\frac{\left(a_{1, n}^{2}+2 a_{2, n} a_{x, n}\right)^{3}}{a_{1, n}^{3}\left(1+a_{2, n}\right)^{3}}=\left(O\left(3^{-n}\right)+2 \cdot \frac{a_{x, n}}{a_{1, n}}\right)^{3}\left(1+O\left(\zeta^{-6^{n}}\right)\right) \\
& =8\left(\frac{a_{x, n}}{a_{1, n}}\right)^{3}\left(1+O\left(3^{-n}\right)\right) .
\end{aligned}
$$

Using the techniques of Lemma 6 we obtain

$$
a_{x, n}=2^{-3 / 2} \beta^{3^{n}} a_{1, n}\left(1+O\left(3^{-n}\right)\right)
$$

for some constant $\beta>1$. Finally this implies that

$$
\frac{a_{2, n+1}}{a_{x, n+1}} \geq \frac{12 a_{2, n}^{4} a_{x, n}^{2}}{\left(a_{1, n}^{2}+2 a_{2, n} a_{x, n}\right)^{3}}=\frac{3}{2} \cdot \frac{a_{2, n}}{a_{x, n}}\left(1+O\left(\beta^{-3^{n}}\right)\right) .
$$

Thus we can reduce the system of recursions to

$$
\begin{aligned}
& a_{0, n+1}=3 a_{1, n}^{2}\left(1+O\left(\zeta^{-6^{n}}\right)\right) \\
& a_{1, n+1}=a_{1, n}^{3} a_{2, n}^{3}\left(1+O\left(\zeta^{-6^{n}}\right)\right), \\
& a_{2, n+1}=a_{2, n}^{6}\left(1+O\left(\left(\frac{2}{3}\right)^{n}\right)\right) \\
& a_{x, n+1}=8 a_{2, n}^{3} a_{x, n}^{3}\left(1+O\left(\beta^{-3^{n}}\right)\right) .
\end{aligned}
$$


From this we easily obtain

$$
\begin{aligned}
& a_{0, n}=3 \beta_{1}^{6^{n} / 3} \beta_{2}^{2 \cdot 3^{n} / 3}\left(1+O\left(\left(\frac{2}{3}\right)^{n}\right)\right), \\
& a_{1, n}=\beta_{1}^{6^{n}} \beta_{2}^{3^{n}}\left(1+O\left(\left(\frac{2}{3}\right)^{n}\right)\right), \\
& a_{2, n}=\beta_{1}^{6^{n}}\left(1+O\left(\left(\frac{2}{3}\right)^{n}\right)\right), \\
& a_{x, n}=2^{-3 / 2} \beta_{1}^{6^{n}} \beta_{3}^{3^{n}}\left(1+O\left(\left(\frac{2}{3}\right)^{n}\right)\right),
\end{aligned}
$$

where $\beta_{1}>1$ and $\beta_{2}, \beta_{3} \in(0,1)$. Since $a_{1, n} \leq a_{x, n}$ it follows that $\beta_{2}<\beta_{3}$. Numerical values of $\beta_{1}, \beta_{2}, \beta_{3}$ are given by

$$
\beta_{1} \approx 1.96936033, \quad \beta_{2} \approx 0.43546557, \quad \beta_{3} \approx 0.92526029 .
$$

We remark that this type of asymptotics always holds when $a_{\nu, n}=o\left(a_{2, n}\right)$ for $\nu \in\{0, v, w, x\}$. In this case the reasoning used in the example above applies, so that

$$
a_{v, n} \approx \beta_{1}^{s^{n}} \beta_{v}^{d_{w}^{n}}, \quad a_{w, n} \approx \beta_{1}^{s^{n}} \beta_{w}^{d_{v}^{n}}, \quad a_{2, n} \approx \beta_{1}^{s^{n}}, \quad a_{x, n} \approx C \beta_{1}^{s^{n}} \beta_{x}^{c^{n}},
$$

where $\beta_{1}>1, \beta_{v}, \beta_{w}, \beta_{x} \in(0,1), C>0, d_{v}=\operatorname{deg}(v), d_{w}=\operatorname{deg}(w)$, and $c$ is the number of edges in a minimal $v$-w cut. The behavior of $a_{0, n}$ is possibly different due to the fact that $G \backslash\{v, w\}$ may be disconnected.

Finally, we conjecture that the number of connected subgraphs exhibit a phase transition: when $\rho>1$, then the asymptotics of Theorem 2 holds, whereas the asymptotics is given by (4) if $\rho<1$.

\section{Acknowledgements}

We would like to thank an anonymous referee for various valuable comments and suggestions. 


\section{References}

[1] M. T. Barlow. Diffusions on fractals. In Lectures on probability theory and statistics (Saint-Flour, 1995), pages 1-121. Springer Verlag, Berlin, 1998.

[2] B. Bollobás. Modern graph theory, volume 184 of Graduate Texts in Mathematics. Springer-Verlag, New York, 1998.

[3] S.-C. Chang and L.-C. Chen. Spanning forests on the Sierpinski gasket. preprint, arXiv:mathph/0612083v1, 2006.

[4] S.-C. Chang and L.-C. Chen. Dimer coverings on the sierpinski gasket with possible vacancies on the outmost vertices. J. Stat. Phys., 131(4):631-650, 2008. arXiv:0711.0573v1.

[5] S.-C. Chang and L.-C. Chen. Dimer-monomer model on the Sierpinski gasket. Physica A: Stat. Mech. Appl., 387(7):1551-1566, 2008. arXiv:cond-mat/0702071v1.

[6] S.-C. Chang, L.-C. Chen, and W.-S. Yang. Spanning trees on the Sierpinski gasket. J. Stat. Phys., 126(3):649-667, 2007. arXiv:cond-mat/0609453v1.

[7] P. G. Doyle and J. L. Snell. Random walks and electric networks, volume 22 of Carus Mathematical Monographs. Mathematical Association of America, Washington, DC, 1984. Also available at arXiv.org as math.PR/0001057.

[8] J. Kigami. Analysis on fractals, volume 143 of Cambridge Tracts in Mathematics. Cambridge University Press, Cambridge, 2001.

[9] G. R. Kirchhoff. Über die Auflösung der Gleichungen, auf welche man bei der Untersuchung der linearen Verteilung galvanischer Ströme geführt wird. Ann. Phys. Chem., 72(4):497-508, 1847. Gesammelte Abhandlungen, Leipzig, 1882.

[10] B. Krön. Growth of self-similar graphs. J. Graph Theory, 45(3):224-239, 2004.

[11] L. A. Malozemov. The difference Laplacian $\Delta$ on the modified Koch curve. Russian J. Math. Phys., 1(4):495-509, 1993.

[12] V. Metz. The short-cut test. J. Funct. Anal., 220(1):118-156, 2005.

[13] A. D. Sokal. The multivariate Tutte polynomial (alias Potts model) for graphs and matroids. In Surveys in combinatorics 2005, volume 327 of London Math. Soc. Lecture Note Ser., pages 173 226. Cambridge Univ. Press, Cambridge, 2005.

[14] E. Teufl and S. Wagner. The number of spanning trees of finite sierpinski graphs. In Fourth Colloquium on Mathematics and Computer Science, volume AG of DMTCS Proceedings, pages 411-414, 2006.

[15] E. Teufl and S. Wagner. Enumeration problems for classes of self-similar graphs. J. Combin. Theory Ser. A, 114(7):1254-1277, 2007.

[16] E. Teufl and S. Wagner. The number of spanning trees in self-similar graphs. preprint, 2008. 
